\title{
DSM 5: Controversial Acceptance and Ongoing Challenges
}

\author{
Shinu Kuriakose* \\ School of Health Professions, New York Institute of Technology, USA \\ *Corresponding author: Shinu Kuriakose, Associate Professor, DHSc. PA-C, School of Health Professions, New York Institute of \\ Technology, USA
}

To Cite This Article: Shinu Kuriakose, DSM 5: Controversial Acceptance and Ongoing Challenges. 2020 - 7(4). AJBSR.MS.ID.001169.

DOI: 10.34297/AJBSR.2020.07.001169.

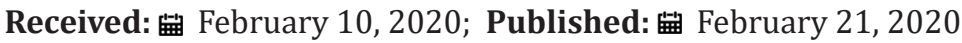

\begin{abstract}
Mental health disorders effect almost $25 \%$ of Americans in a given year and is the leading cause of disability. These disorders can cause substantial detrimental changes in society due to lack of proper screening principles and comprehensive care. Disparities in health, education, employment, wealth and socio-economic standings are quite apparent in the psychiatric population. The Diagnostic and Statistical Manual of the American Psychiatric Association has published a book, which organized mental health pathologies into distinct groups to further standardize the way these illnesses can be categorized. The latest version of this manual, the DSM 5, was published in 2013 and it includes specific changes compared to the DSM IV. There continues to be still criticism aimed at the DSM 5 due its focus on symptomology instead of biological underpinnings of mental illness. Ultimately, the DSM 5 is an effort to further standardize diagnosis of mental illness among all populations with further reduction in mental health disparities.
\end{abstract}

Keywords: Mental health; DSM 5; Health disparities; American Psychiatric Association

\section{Introduction}

One in four Americans over the age of 18 suffer from a diagnosable mental illness and it is the leading cause of disability in the United States [1]. The prevalence of lifetime mental health disorders worldwide is between $18-36 \%$ while the annual prevalence is $10-20 \%$ [2]. The field of psychiatry has historically suffered from inaccurate diagnosis of specific psychological disorders, as different societies often view psychiatric symptoms from a cultural viewpoint. What is considered the cultural norm in one society might be seen as deviant pathology in another. In an attempt to bridge this gap and provide more standardized care for patients, the American Psychiatric Association (APA) published the Diagnostic and Statistical Manual of Mental Disorders (DSM) in 1952. The DSM has often been referred to as the "bible" of psychiatry. The manual organizes psychiatric disorders into specific categories with precise characteristics and symptoms; a process, which helps, standardize psychiatric diagnosis all over the world, especially with psychiatry at times being so subjective. This approach also helps different researchers publish their psychiatric findings in journals using the same standardized scales, which facilitates a review and duplication of their findings [3].
However, the approach to focus on mental conditions and the associated symptoms has been cited as one of the major deficiencies in the DSM. The deficiency is due to the DSM's focus on symptomatology, not the underlying reasons of the disorder. "A DSM-informed psychiatric diagnosis is based mainly on selfreports of feelings and experiences by patients with diverse backgrounds and on clinicians' understanding of psychiatric terms or observation of behavior. Such subjective impressions of complex phenomena can lead to diagnostic inconsistencies across patients and practitioners" [4]. Another criticism of the DSM has been that it takes current social mores into account, which might not reflect societal beliefs in the future. For example, homosexuality was listed as a pathological sociopathic personality trait from 1952 to 1974 and believed to be caused by a traumatic parent-child relationship. This theory was thoroughly refuted after further research.

The DSM has also been consistently evaluated and has undergone changes over the years; the most recent being in 2013 with the Diagnostic and Statistical Manual of Mental Disorders, Fifth Edition (DSM 5). According to the APA, a task force of 160 worldrenowned clinicians and researchers reviewed various scientific 
breakthroughs and papers over the last decade, resulting in the updated version. "Many of the changes in DSM-5 were made to better characterize symptoms and behaviors of groups of people who are currently seeking clinical help but whose symptoms are not well defined by DSM-IV (meaning they are less likely to have access to treatment)" [5] . The last update to the DSM was in 1994 with the creation of DSM 4; a change 14 years in the making [5].

\section{Review of Literature}

\section{Major changes from DSM IV to DSM 5}

One of the fundamental changes was from the Roman numeral numbering system (IV) to Arabic numbering (5). The decision was made to make the DSM more nimble and responsive to constant changes in definitions and symptomology in psychiatry and the subsequent versions would be then called DSM 5.1, 5.2 etc; a process repeated until a complete new revision was warranted [5]. Additionally, the multi-axial conditions coding has been changed by incorporating the first three axes into one list, which would include not only all psychiatric conditions, including personality disorders and mental retardation, but also general medical conditions [5]. Psychological or environmental factors contributing to the patient's mental pathology can further be coded using International Classification of Diseases (ICD) methodology and noted in the medical chart as additional information [5]. The Global Assessment of Functioning has been omitted from the DSM 5 due to the inherent unreliability of assigning a single number to a patient concerning their assessment and symptom severity; rather, clinicians are encouraged to evaluate patient's condition and severity of suicidal or homicidal risk using several established scales, which are readily available [5].

\section{Specific domain revisions in the DSM 5}

Neurodevelopmental disorders: The term "mental retardation" has been changed to "intellectual disability", therefore reflecting the deficits in cognitive ability during the developmental phase of childhood; this new phrase has being more commonly used by both providers and the public in the past several years [5]. The emphasis has been more focused on adaptive learning rather than an intelligence quotient (IQ) number. Among communication disorders, stuttering has been changed to language fluency disorder to reflect the pathology behind this condition. A new condition, social communication disorder, has been added to reflect both verbal and non-verbal persistent deficits in communication in the social setting. Language disorder diagnosis now encompasses both expressive and mixed receptive-expressive disorders while the term phonological disorder has been changed to speech sound disorder; a term which lends itself to more of a explanatory understanding of the pathology. Furthermore, autism spectrum disorder in DSM 5 encompasses the four distinct pathologies in DSM- IV: (a) the previous DSM-IV autistic disorder (autism), (b)
Asperger's disorder, (c) childhood disintegrative disorder, and (d) pervasive developmental disorder not otherwise specified [6]. This change-reflecting consensus that all four subgroups was present in an autistic patient and the severity of this illness was more reflective of the condition. Lastly, the onset criteria used to diagnose Attention Deficit/Hyperactivity Disorder (ADHD) has been changed from symptoms occurring before age seven to hyperactive impulses before age 12 [7]. Reading disorders (dyslexia), mathematical disorders (dyscalculia), writing disorders have all been grouped into specific learning disorders, as there is greater recognition of learning disorders occurring together.

Schizophrenia and other psychotic disorders: Schizophrenia, per the DSM 5 (2013), will now need at least two Criteria A symptoms (also called positive/first rank symptoms) for diagnosis: (a) delusions, (b) hallucinations, or (c) disorganized speech. DSM 5 eliminates the need for a person to have a "bizarre" delusion due to poor reliability between describing a bizarre versus a non-bizarre delusion (APA, 2018). Furthermore, the subtypes of schizophrenia, such as (a) paranoid, (b) disorganized, (c) catatonic, and (d) undifferentiated have been eliminated in the new DSM due to poor validity and limited use in treatment methods. In an effort to improve the validity and reliability of diagnosing schizoaffective disorder, DSM 5 requires that a major mood component be present during the majority of the disorder, after Criteria A has been confirmed. Delusional disorder has now been updated to also include "bizarre delusions", in addition to the "non-bizarre delusions".

Mood and anxiety disorders: There has been an emphasis of increased activity and energy in Bipolar disorder in addition to mood lability to ensure better predictability and earlier detection clinically [5]. Dysthymia has been labeled as persistent depressive disorder, which reflects the longitudinal nature of this condition. The exclusion of a diagnosis of "major depression" within two months of losing a loved one, which was initially labeled as bereavement, has been cast aside. The faulty reasoning that bereavement should only last 2 months has been refuted by multiple clinical studies and thus a person prone to bereavement has a higher likelihood of being diagnosed with major depression [5]. Social anxiety and agoraphobia have also had requirements changes that include a person feeling that their anxiety is excessive or unreasonable which is out of proportion to the situation.

Feeding disorders: Anorexia Nervosa has the requirement of amenorrhea eliminated due to greater recognition that a substantial number of men suffer from this. Bulimia has had a change whereby a single binge-eating episode a week can meet the criteria for this illness whereas previously two episodes a week were needed [5].

Substance related disorders: Gambling disorder, cannabis withdrawal, and caffeine withdrawal are new additions to the DSM 5, despite the difference between substance abuse and substance dependence has been eliminated [5]. 
Neurocognitive disorders: Major Neurocognitive disorder has taken the place of dementia while an addition of mild neurocognitive disorder has been added to diagnose milder cognitive deficits in patients [5].

\section{Implication for clinical practice}

One of the major concerns with the new DSM was the challenges in billing practices and reimbursement. The APA has mitigated this obstacle by having the DSM 5 fully compatible with the codes with the current ICD 9 and upcoming ICD 10; a transition, which should assure consistent, thorough continuity of care. Currently, it is expected that the ICD 10 will be fully functional on October 2015 .

\section{Conclusion}

Over the last two decades, there has been a paradigm shift in the field of psychiatry; the emphasis of neurodevelopment changes causing symptoms and the renewed focus on illnesses falling on a spectrum rather than a vacuum. Furthermore, there is added emphasis to evaluate and use biological causes of mental illness to define the pathology, versus relying just on symptoms. The DSM 5 also strives to make clinicians understand that multiple psychiatric illnesses can present atypically and this subtle change must be taken into account during treatment regimens. Additionally, DSM
5 is more consistent with ICD 10 coding to better align itself in accurate reimbursement rates with satisfactory outcomes; both patient centered and financial.

\section{References}

1. National Institute of Mental Health (2018) The Numbers Count: Mental Disorders in America.

2. Kessler, Aguilar-Gaxiola S, Alonso J, Chatterji S, Lee S, et al. (2009) The global burden of mental disorders: An update from the WHO World Mental Health (WMH) Surveys. Epidemiol Psichiatr Soc 18(1): 23-33.

3. American Psychiatric Association (2018) DSM-5 Development.

4. Casey BJ, Craddock N, Cuthbert BN, Hyman SE, Lee FS, et al. (2013) DSM5 and RDoC: progress in psychiatry research? Nat Rev Neurosci 14(11): 810-814

5. American Psychiatric Association (2018) Insurance implications of DSM

6. Kulage KM, Smaldone AM, Cohn EG (2018) How will DSM-5 affect Autism Diagnosis? A Systematic Literature Review and Meta-Analysis. J Autism Dev Disord 44(8): 1919-1932.

7. Al-Yagon M, Cavendish W, Cornoldi C, Fawcett AJ, Grünke M, et al. (2013) The proposed changes for DSM-5 for SLD and ADHD: International perspectives--Australia, Germany Greece, India, Israel, Italy, Spain, Taiwan, United Kingdom, and United States. J Learn Disabil 46(1): 58-72. 